\title{
Ultrafast dynamical diffraction wavefronts in strained Si imagined with Tele-ptychography
}

\author{
Angel Rodriguez-Fernandez ${ }^{1}$, Ana Diaz ${ }^{2}$, Anand H. S. lyer ${ }^{3}$, Mariana Verezhak ${ }^{2}$, Klaus Wakonig ${ }^{2}$, \\ Magnus H. Colliander ${ }^{3}$, Dina Carbone ${ }^{4}$
}

\author{
${ }^{1}$ Eu XFEL GmbH, Schenefeld, Germany; \\ ${ }^{2}$ Paul Scherrer Institute, Villigen PSI, Switzerland; \\ ${ }^{3}$ Chalmers University of Technology, Gothenburg, Sweden; \\ ${ }^{4} \mathrm{MAX} I V$, Lund University, Lund, Sweden; \\ angel.rodriguez-fernandez@xfel.eu
}

Dynamical diffraction effects, also known as echoes, produced in thin crystals in both forward and diffracted directions are of highest importance for X-ray optics at ultrafast sources, as XFELs, and for the study of ultrafast phenomena in micron-sized single crystals. These echoes present delays of few fs between each other and the transmitted beam (similar as it happens with sound echoes, but in this case of electromagnetic nature and therefore with the speed of light). The delay relates to a displacement of the monochromatic diffracted beams in the transverse direction to the X-ray beam propagation $[1,2]$. Such echoes are used in self-seeding forward monochromators at hard xFELs.

We would like to present our work performed at NanoMAX, MAX IV laboratory, Sweden, in which we image the dynamical diffraction wavefront from a $100 \mu \mathrm{m}$ thick Si wafer [3]. The work uses the full coherence and high flux of NanoMAX, together with the technique known as tele-ptychography [4], to image the forward diffracted wavefront at a pinhole located 3 mm downstream the sample. As presented in figure 1, the data collected is reconstructed using a ptychography algorithm in the pinhole plane, obtaining amplitude and phase of the wavefront. The wavefront is propagated back to the focus where, combined to the small size of the X-ray beam provided by NanoMAX, provides a high- resolution $(55 \mathrm{~nm})$ image for the detection of forward diffracted echoes.

The work underline how this effect must be taken into account in the imaging and study of samples with thickness of the order of the X-ray extinction length. We also show that a strain induced in the surface can modulate the temporal delay of the dynamical diffraction waves as presented in the second figure attached. All the work is accompanied with the simulation of the effect using a selfwritten code, that can be used to model both temporal and static strains in single crystal samples, as well as in micro-pillars in which these dynamical effects are also present [5].
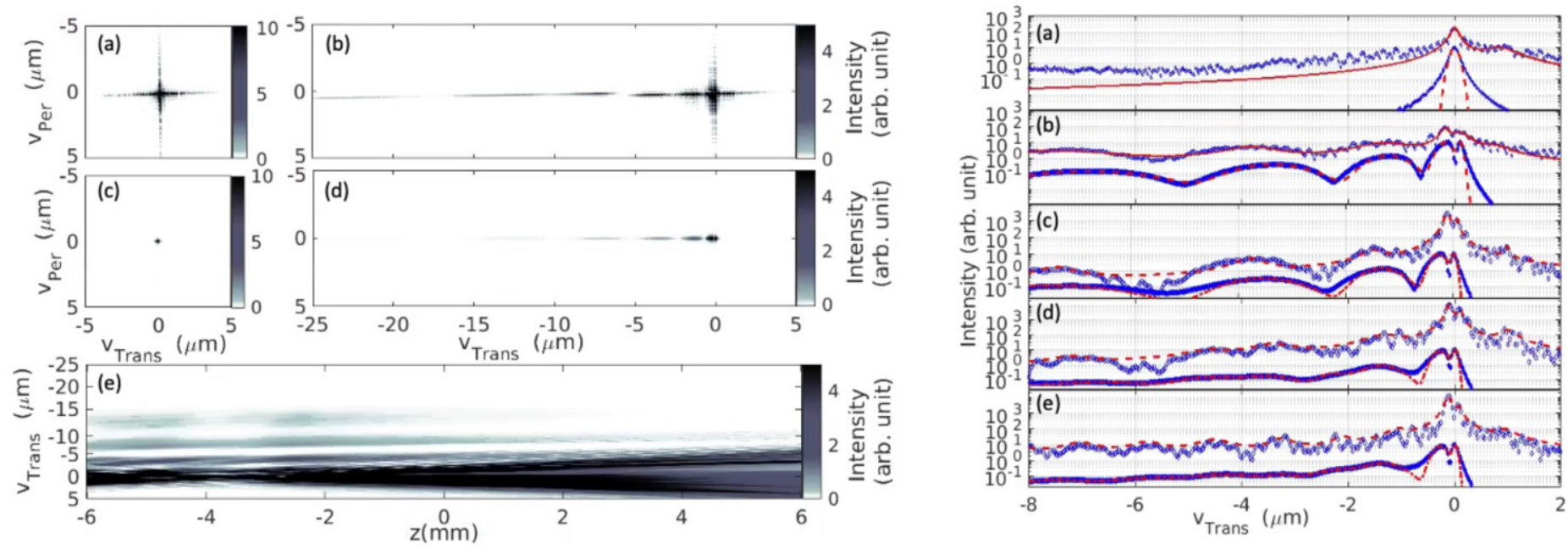

Figure 1. Ptychographic reconstruction of the forward beam propagated to the focus $(100 \mathrm{~nm})$ at NanoMAX of a $100 \mu \mathrm{m}$ thin Si crystal (a) $1^{\circ}$ away (b) at the asymmetric (111) diffraction condition at $8 \mathrm{keV}$. Simulations of the forward beam performed using a code based in dynamical diffraction theory (c) $1^{\circ}$ away and (d) at the symmetric (111) diffraction condition at $8 \mathrm{keV}$. (e) Propagation of the forward ptychographic reconstructed signal along the plane defined by the beam propagation and the transverse direction to diffraction.
Figure 2. Projection simulations along the transverse direction to diffraction of the forward beam (open dots) ptychographic reconstructions and (fill dots) simulations for a non-strained Si $100 \mu \mathrm{m}$ thin crystal (a) $1^{\circ}$ away of the diffraction condition, (b) at the asymmetric (111) diffraction condition at $8 \mathrm{keV}$, and a nano-indented $\mathrm{Si}$ $100 \mu \mathrm{m}$ thin crystal at the asymmetric (111) diffraction condition at 8 $\mathrm{keV}$ in three locations (c) 1000 away from indents, (d) at $25 \mathrm{mN}$ indentation and at $75 \mathrm{mN}$ indentation. 
[1] A. Rodriguez-Fernandez et al., ActaCryst. A74, 75 (2018)

[2] Y. Shvydko and R. Lindberg, Phys. Rev. ST Accel.Beams15, 100702 (2012)

[3] A. Rodriguez-Fernandez et al., Imaging ultrafast dynamical diffraction wavefronts in strained Si with coherent X-rays arXiv:2012.08893 (2020)

[4] E. H. R. Tsai et al, Optics Express 34 (2016) 6441; [5] M. Verezhak et al. arXiv:2012.03299 (2020)

Keywords: Coherence, X-rays, Ultrafast, dynamical, diffraction 